\title{
LE REGARD DES JOURNALISTES ET DES POLITIQUES SUR LES ÉMISSIONS POLITIQUES
}

\author{
Joëlle Desterbecq ${ }^{1}$
}

Une série d'entretiens semi-directifs ont été menés auprès du personnel politique mais aussi auprès des journalites-animateurs et des instances de direction des deux principales chaînes diffusées en Communauté française. Le volet d'enquête auprès de la classe politique est composé de deux sections. Aux interviews «face-to-face », nécessitant de travailler sur des groupes restreints, s'ajoute une section de recherche par questionnaire. Un exemplaire en six feuillets a été adressé à tous les parlementaires de la Communauté française. Les répondants se différencient donc par leur degré d'exposition médiatique. À cet égard les enquêtes par entretiens et questionnaires se complètent. Le premier type donne la parole aux personnalités médiatiques alors que le second fait apparaître des acteurs qui interviennent ponctuellement sur la scène médiatique.

\section{Les éditeurs de services}

\section{Les débats dominicaux : glissement générique ?}

Comme précisé plus haut, Mise au point et Controverse traitent de sujets similaires, souvent avec les mêmes invités et selon une dyna-

1 Joëlle Desterbecq est chercheuse à l'Observatoire du récit médiatique (ORM) au Département de communication de l'UCL.

Recherches en communication, $\mathrm{n}^{\circ} 24$ (2005). 
mique identique. Les deux chaînes décrivent, à leur égard, une évolution analogue : ces rendez-vous dominicaux auraient au fil du temps élargi le panel des sujets traités afin d'y ancrer les faits ou sujets dits « de société ». Nos interlocuteurs justifient cette évolution par une demande sociale issue, entre autres, de l'affaire Dutroux et qui s'est concrétisée dans la «marche blanche».

Ainsi, l'introduction des sujets de société va de pair avec celle des « citoyens anonymes » convoqués pour illustrer un phénomène. Ces citoyens ordinaires ont vocation à concrétiser, au départ de leur expérience vécue ou des comportements qu'ils ont expérimentés, les tenants et aboutissants de certains dossiers. Ils sont conviés par les journalistes en tant que «témoins » d'un phénomène : Cette introduction n'est pas interprétée de la même manière par les deux chaînes en termes d'impact sur la classification générique de leur programme dominical. Ainsi, si RTL-TVi revendique un qualificatif de «débat de société » pour son émission Controverse, la RTBF n'envisage pas que l'on puisse extraire son programme du champ politique au sens strict. Sur la chaîne privée, la présence de multiples témoins a pour finalité explicite et revendiquée la constitution d'un plateau le plus éclectique possible en vue de générer un conflit, une controverse, en vue d'appuyer cette « sémiologie des oppositions ».

Dynamique des altercations organisées, introduction de thèmes dits de société et de citoyens anonymes témoignant de leur expérience personnelle, mettent en exergue l'importation de procédés fondateurs des talk-shows au sein des débats dominicaux de la Communauté française. Ces évolutions témoigneraient ainsi d'un premier pas vers un glissement générique. Les prestataires de services s'en défendent. La RTBF refuse tout autre qualificatif que celui de «politique » pour son émission du dimanche, RTL, quant à elle, introduit des nuances assez subtiles pour récuser le qualificatif de talk-show.

\section{Le mélange des genres}

Les chaînes de télévision ont présenté au cours de la campagne électorale de 2004 des émissions à la frontière de plusieurs genres : Face à l'électeur et Pour qui voter? sur RTL; Face à face et Destination élections à la RTBF. Le premier type d'arguments justifiant ce choix renvoie à la monotonie qui entourerait la parole politique. Les émissions à la frontière de plusieurs genres permettraient de contrecarrer les baisses d'audience qui iraient souvent de pair avec la diffusion de programmes 
à contenu politique, en mettant en œuvre des programmes accrocheurs dans la forme. Sur la chaîne publique, même si les objectifs semblent être identiques, les motivations sont présentées de façon plus nuancée : " c'est une volonté d'accroche, comme on dit, dans la confection d'un produit destiné à publication », ou encore : «nous devons tenir compte d'une situation, qui est l'attitude du téléspectateur. Le téléspectateur est confronté à des offres de stimulus ou de stimuli visuels nombreux et variés. Et il a une tendance naturelle à se diriger vers ce qui capte son attention ». Offrir un programme qui capte l'attention face à un panel de stimuli semble bien constituer une préoccupation en termes d'audimat, il s'agit de prévenir le zapping du téléspectateur. Pourtant, la chaîne tend à relativiser ce paramètre en insistant sur ses missions de service public.

Les éditeurs de services laissent entrevoir une convergence entre l'intérêt de la chaîne et celui de ses téléspectateurs. Grâce à l'hybridation des genres, tout le monde serait gagnant: la télévision préviendrait la «dévastation » des audiences, et les téléspectateurs réinvestiraient l'espace public. Ainsi, l'interpénétration générique permettrait de ramener dans la sphère publique de libre expression les citoyens qui tendraient à s'en éloigner, par désintérêt ou par incompréhension de la chose publique, en leur proposant un concept novateur et attrayant. La télévision affirme presque faire œuvre de salut public en réinstaurant un contact entre les mandataires publics et une large frange de citoyensélecteurs.

Les émissions hybrides tendent à augmenter le degré d'intervention de l'instance médiatique sur le format du message. Le média définit ou code les règles d'émission du message politique qui doit se fondre dans ce canevas prédéterminé. Ainsi, dans l'émission L'invité, le représentant politique doit se soumettre à une série de questions types telles que le « Podium», les «Cotes», le « Oui ou non» etc., qui tendent non seulement à baliser le message mais aussi à le simplifier. Le dispositif ainsi constitué favoriserait, aux dires de ses concepteurs, la clarté du message politique. La forme ludique du programme tendrait donc à prévenir, voire à déjouer les stratégies de communication politique.

Notons que l'aspect ludique ne va pas toujours de pair avec le contournement de la communication politique. En effet, la dimension de jeu peut être récupérée à des fins de stratégie politique. La RTBF en a fait l'expérience avec l'émission C'est ma voix, débat à l'aveugle présenté au cours de la campagne électorale de 2003 : «au moins un attaché de presse avait avoué très clairement que l'objectif, c'était de 
brouiller l'image de la personne qui était de l'autre côté en donnant un nom totalement absurde. C'est donc pour ça qu'on a arrêté et c'est devenu Destination Elections ».

$\mathrm{Au}$ moyen de concepts tels que Destination élections ou Face à l'électeur, la télévision vise à s'arroger un meilleur contrôle sur le déroulement des programmes en favorisant l'éviction d'un message politique consensuel. En effet, l'objectif est d'introduire des contradicteurs extérieurs au schéma de communication préétabli par les représentants politiques. Le mélange des genres est présenté ici comme un indice du rapport de force qui se joue entre la classe politique et l'instance médiatique pour le contrôle du contenu du message émis à l'attention du spectateur. L'hybridation aurait vocation à contourner l'art du discours soigneusement contrôlé pour favoriser le «parler-vrai ».

Résorber le fossé qui se crée davantage entre le citoyen et ses représentants lorsque ces derniers débattent dans un microcosme fermé, en usant d'un langage pas toujours compréhensible par tout un chacun, est une motivation supplémentaire qui aurait présidé à la création de Destination élections. Ainsi, cette émission, qui sort des schémas classiques de représentation du politique, affiche pourtant comme ambition de renouer avec la préoccupation initiale de la politique, à savoir : la vie de la Cité. Une fois encore, un contact entre les citoyens et leurs représentants est rétabli par la médiation de la télévision. Cette fonction phatique, de contact, est mise en œuvre assez pragmatiquement : en confrontant le mandataire public à la vie de la cité. Ainsi, le principe de l'émission nous a été décrit comme un test en vue d'observer d'une part, si le représentant politique est ou non en phase avec les préoccupations de la Cité, et d'autre part, s'il parvient à mettre en œuvre ce qu'il préconise pour son organisation. L'émission «teste » pour le citoyen, elle a une visée «facilitative», en vue de lui permettre de faire son «shopping électoral».

$\mathrm{Au}$ vu des arguments énoncés par les éditeurs de services, l'interpénétration générique serait tout bénéfice pour le citoyen-téléspectateur. Elle rend le message politique plus vivant, plus attractif et par voie de conséquence, ramène vers l'espace public une partie de citoyens susceptibles de se désintéresser de ce qui s'y dit. Elle tend à contourner les possibilités de communication stratégique en poussant à la clarté du message, au "parler-vrai ». Elle résorbe le sillon qui se creuse entre les mandataires et ceux qui les mandatent. Bref, l'interpénétration des genres, mise en œuvre par les chaînes de télévision, nous est présentée comme un palliatif au contact qui ne se ferait plus spon- 
tanément entre les citoyens et les élus - ou plus généralement entre les citoyens et la chose publique. À cet égard, il nous semble que la télévision se présente ici comme une instance médiatrice ou «facilitative » : elle crée un nouveau lien. Elle tend à diversifier l'offre de programmes en vue de mettre en contact des individus qui vivraient complètement déconnectés sans sa médiation.

Le ludique est présenté exclusivement comme un outil au service du contenu de l'émission. La logique qui préside à la conduite des émissions n'est pas un évitement ou un contournement de la chose publique. Les programmes hybrides ne sont pas à confondre avec ceux de divertissement, car le regard qu'ils portent sur le politique est précisément politique. Cette trame de fond préviendrait tout dérapage vers des formats de pur spectacle. Le ludique est simplement apparenté à la «touche peps » du programme. C'est un habillage qui modernise le produit et, aux yeux de ses concepteurs, rend son contenu moins monotone.

Cependant, ce raisonnement porte en lui-même ses limites. Jusqu' où peut-on considérer que le ludique ne constitue qu'un habillage du contenu ? À partir de quand le ludique - ou le fictionnel - prennentils le dessus ? Une fois encore, nos interlocuteurs sont unanimes sur un point. Il convient de ne pas altérer la nature du message, voire l'intégrité du monde politique. Néanmoins, cette unanimité est évanescente. Elle s'envole peu à peu lorsqu'il est question de déterminer ce qu'est un message altéré ou une intégrité mise à mal. Précisons d'emblée que les lignes de démarcation qui se figent ne relèvent pas d'un dualisme privé-public. En effet, nous avons observé au sein d'une même unité de programmes de la chaine de service public des divergences fondamentales sur la question du point de rupture à partir duquel le contenu est dénaturé. La cristallisation des antagonismes s'est opérée au départ de l'évocation du programme Vote for me diffusé par la télévision privée britannique au cours de la dernière campagne électorale. Pour les uns, de tels formats qui allient politique et télé-réalité peuvent poser question quant à la responsabilité politique de la chaîne. En effet, des programmes tels que celui de ITN ont mis en exergue le décalage existant entre les légitimités cathodique et élective. Pour les autres, ce type d'émission, pour peu que l'on tente d'en contrôler les dérapages, possède incontestablement des vertus pédagogiques. Notre interlocuteur a d'ailleurs surenchéri dans le mélange des genres en vantant les mérites du « docufiction » pour la compréhension des coulisses de la vie politique.

De telles oppositions témoignent du fait que la réflexion sur des formats politiques toujours plus proches des modes d'énonciation fictif 
et ludique est inévitable et n' «épargne » aucune chaîne. À cet égard, il conviendrait de sortir des oppositions manichéennes selon lesquelles de tels produits ne pourraient s'ancrer que parmi l'offre des chaînes privées.

\section{La personnalisation}

Les représentants politiques interviewés se sont accordés à dire que la frontière vie privée - vie publique est relativement peu perméable à l'exception notoire de l'émission L'invité, diffusée sur RTL. Quant aux programmes de la campagne électorale, quatre d'entre eux tendent à personnaliser un tant soi peu les propos des représentants politiques. Nous avons demandé aux chaînes quel est le but poursuivi par ces mises au jour de la vie privée.

RTL, un peu plus coutumière que la RTBF de la personnalisation des programmes politiques, se défend d'évoquer la vie privée des personnalités publiques : «les limites, c'est clairement la vie privée »; «Je ne suis pas d'accord. Les questions sont toujours politiques au sens large ». En fait, nous observons au fil des entretiens que nos interlocuteurs de la chaîne privée constituent implicitement une «échelle » de personnalisation. À l'extrémité gauche de cette échelle (moins de personnalisation), nous retrouverions les questions qui sollicitent une prise de position personnelle sur un thème d'actualité - voire une anecdote de la vie politique. Il s'agit en quelque sorte de questions privées contextualisées. Viennent ensuite les questions qui cherchent à mettre au jour une vision du monde philosophique, religieuse, etc. Au-delà de ce seuil, la frontière de la vie privée serait franchie. Néanmoins, une distinction est opérée par nos interlocuteurs entre le registre de la vie privée «au second degré » et le registre privé au sens strict, situé à l'extrémité droite de l'échelle.

Aux dires des interviewés, les questions posées aux mandataires publics ne se situeraient en aucun cas à l'extrémité droite de l'échelle. Cela ne signifie donc pas qu'il n'existe pas un certain degré de personnalisation des questions. Celle-ci aurait d'ailleurs trois vocations. Premièrement, il s'agirait d' "humaniser» le personnel politique «ce sont d'abord des femmes et des hommes comme les autres, qui travaillent, qui ont une mission, un boulot: des problématiques d'humain. Je ne vois pas d'autres types d'espèces dont ils font partie »- en vue de ne pas perdre le contact entre ce dernier et leurs concitoyens. Deuxièmement, dans la mesure où les idées politiques sont forgées par 
la vie et l'expérience, découvrir un tant soit peu ces paramètres augmenterait la connaissance que le citoyen a de ses représentants politiques et partant, de leurs idées. Il semble intéressant de préciser que la problématique de «l'humain » est un argument employé par le personnel politique lui-même en vue de justifier une participation aux émissions qui dépassent le cadre politique au sens strict. Troisièmement, ce registre d'interview permettrait d'atteindre un public plus vaste que celui qui suit les débats politiques classiques. Il y a donc un intérêt incontestable en termes d'audience.

Sur la chaîne publique, Destination élections et le Face à face électoral constituent les deux seuls programmes politiques qui personnalisent un tant soit peu les interviews. En effet, la première émission semble laisser une marge aux représentants politiques qui souhaitent mettre en évidence quelques aspects plus personnels. Charles Picqué, en quête d'emploi à l'ORBEM, apprendra au téléspectateur ses talents de cuisinier et de jardinier. Le choix de laisser apparaître - pour partie - des personnes plutôt que des politiques est motivé au moyen de considérations proches de celles développées par la chaîne privée : "La personnalité d'un homme politique n'est pas quelque chose de neutre dans le choix des électeurs. Un choix électoral n'est pas uniquement quelque chose qui se fait sur papier : "Ah oui, pour les impôts, il va faire ci, etc. «. Il y a ce capital sympathie que l'homme politique parvient plus ou moins à faire passer et qui fait partie du jeu démocratique ». Le capital sympathie, l'humain, est la clé du contact entre le citoyen et un représentant politique qui tend à apparaître comme son miroir.

\section{Du plus moderne au plus traditionnel : le débat électoral}

En contrepoint des émissions qui optent pour un genre hybride ou une personnalisation des questions, les chaînes conservent des rendezvous plus traditionnels dans leur forme tels que les débats électoraux ou les soirées électorales. En effet, quelle que soit la réflexion sur les nouveaux formats à mettre en œuvre pour drainer un public plus vaste, le débat - sous son acception la plus classique d'échange argumenté entre mandataires de divers partis - demeure un pilier aux allures inébranlables dans la programmation des chaînes. Les programmes mixtes ou «personnalisants » représenteraient donc une offre exclusivement complémentaire aux formats classiques et non une offre de substitution. La volonté des prestataires de services ne semble guère être celle d'inverser complètement le rapport de force entre les genres 
télévisuels représentant le politique, mais bien de proposer différentes alternatives au téléspectateur.

Cette conception de l'équilibre marque peut-être encore davantage la chaîne de service public. En effet, alors qu'une réflexion y est menée en profondeur sur les formats à proposer au téléspectateur dans un avenir proche, certains considèrent que toute introduction du mode d'énonciation ludique dans un programme politique se résume à faire passer l'accessoire avant l'essentiel. L'un de nos interlocuteurs soutient une argumentation de ce type. Fort de revendiquer son appartenance à "une vague traditionaliste », il considère que l'originalité d'un programme politique doit émaner d'une part, de la force du journaliste à ne pas le rendre ennuyeux, et d'autre part, de son interactivité avec le citoyen-téléspectateur.

Si certains considèrent que la forme ludique d'un programme doit permettre de favoriser le «parler-vrai », notre interlocuteur estime qu'il existe d'autres moyens pour atteindre cette fin : "C'est au journaliste à faire en sorte que ça n'ait pas un côté convenu : il y a toujours moyen de sortir du programme des partis politiques en faisant référence à ce qui a été leur action ». Un programme politique ne pourrait s'accommoder simultanément de la recherche de l'échange argumenté et du jeu. Et si la RTBF ne doit pas devenir « la chaîne de l'austère », il faut néanmoins dissocier ludique et politique.

Cette réflexion atteste, d'une part, que la chaîne ne semble par avoir de position commune quant à l'attitude à adopter face à l'hybridation des genres, et d'autre part, que l'offre de programmes mixtes ne semble pas sur le point d'évincer les rendez-vous traditionnels car la chaîne doit immanquablement composer avec les différentes forces en présence.

\section{Les entretiens avec le personnel politique}

\section{L'offre de programmes}

L'objectif de l'analyse étant de découvrir les représentations médiatiques des personnalités interviewées, nous leur avons demandé de déterminer quelles émissions diffusées en Communauté française elles qualifieraient de politiques. $66.6 \%$ des interviewés citent spontanément Mise au point contre $62.8 \%$ pour Controverse qui, selon certains, « s'écarte de sa mission politique »; $25 \%$ évoquent spontanément $L$ 'invité et enfin $12.5 \%$ parlent des JT et des magazines. Les inter- 
viewés procèdent d'emblée à une distinction entre le temps électoral et le temps « usuel » qui constitue le cadre de représentation du politique. Temps particulier en raison des balises juridiques qui le cloisonnent, le temps électoral comporte un certain nombre d'émissions qui se rapprocheraient davantage de leur «idéal ». En effet, deux axes majeurs se dégagent de leur définition d'une émission politique : une émission politique implique un positionnement partisan ; une émission politique impose qu'il y ait un débat d'idées. Celui-ci comporte deux indicateurs : l'existence d'une confrontation - au sens de comparaison et non de conflit - et celle d'une temporalité - inscription dans la durée.

Tous les mandataires publics qui ont avancé la première définition considèrent que les débats dominicaux diffusés sur les deux principales chaînes francophones s'intègrent dans ce cadre définitionnel et peuvent dès lors être qualifiés d'émissions politiques. En revanche, lorsque ces dernières sont assimilées à un débat d'idées, Mise au point et Controverse sont automatiquement exclus du cadre de représentation politique. Les personnalités interviewées concluent dans ce cas de figure qu'il n'existe «pas vraiment » d'émission politique en Communauté française. Ils sont $29 \%$ à tenir cette opinion. L'absence d'émission politique est justifiée dans ce cas par l'inexistence de débat d'idées permettant d'aborder en profondeur une question après en avoir expliqué les différentes facettes. À titre indicatif, l'un de ces mandataires qualifie les débats dominicaux proposés par les deux chaînes de «divertissement politique ».

Certains hommes publics optent pour une définition restrictive de l'émission politique en introduisant des critères d'exclusion :

- Un programme purement politique n'est pas une émission de reportage, un journal télévisé, une émission de société. Il doit avant tout être tourné vers la vie politique du pays, des différentes institutions, de l'Europe, du monde.

- C'est une émission qui porte sur des enjeux. Ils peuvent être de nature économique commentée, de nature sociologique - la correction des inégalités - toujours sur base de l'action, on n'est pas pour de l'analyse.

D'autres, en revanche, élargissent le cadre de représentation usuel du politique en optant pour des définitions qui intègrent les JT et les émissions d'enquête ou de reportage : 
- Une émission politique est un programme « consacré spécifiquement à un domaine où il y a des responsabilités importantes $d u$ politique, style les émissions que Defossé peut faire».

- «Une émission politique c'est une émission au cours de laquelle on parle de sujets politiques.

- C'est une émission qui doit capter "l'intérêt du citoyen ».

- Enfin, certains distinguent le terme politique au sens strict et au sens large. Au sens large, "la politique, c'est la vie de la cité. Donc si je veux concevoir la vie de la cité, tout peut concerner la vie de la cité. C'est pour cela que je vous dis que la vie de la cité au sens large, la res publica, ça peut être un magazine comme Actuel par exemple ou Tout ça ne nous rendra pas le Congo ».

Néanmoins, les émissions incluses dans le cadre de représentation du politique par ces dernières définitions se bornent à graviter autour du noyau dur constitué des débats dominicaux.

\section{Appréciation des programmes en Communauté française}

Lorsqu'on observe l'appréciation des programmes offerts, on note une étonnante convergence entre les deux catégories d'interlocuteurs. En effet, le profil de l'interviewé ne semble pas jouer à l'égard de certaines réserves formulées envers l'offre de programmes. Premièrement, 96\% des mandataires interrogés mettent en cause le nombre jugé trop élevé de protagonistes invités à prendre part aux débats dominicaux. Il en résulterait une diminution considérable du temps de parole imparti à chaque intervenant avec pour conséquence d'une part l'obligation de résumer sa pensée en slogans simplistes et sans nuances et d'autre part, une configuration du débat sous forme de joute. Deuxièmement, une propriété inhérente à la culture médiatique, à savoir sa logique de flux, semble déteindre sur l'appréciation globale du contenu des programmes proposés. Les commentaires font référence à un chassé-croisé des contenus en vertu duquel une information balaie l'autre selon les aléas de l'actualité, mettant aussi en exergue une circulation de l'information où la télévision se sent obligée de relancer les thématiques abordées durant la semaine dans la presse quotidienne.

La troisième remarque concernant l'offre de programmes est connexe à la précédente. Ces sujets qui se chassent l'un l'autre favoriseraient la superficialité des contenus. Aussi, ce dont manqueraient les émissions francophones, c'est une mise en perspective des thèmes 
abordés. Celle-ci doit être envisagée sous deux acceptions. La première est pédagogique : il s'agit de présenter au téléspectateur les différentes faces du problème que l'on souhaiterait développer. La seconde est historique puisqu'il conviendrait, aux dires de certains interlocuteurs, de resituer l'histoire des positionnements politiques sur un sujet déterminé.

La question de la couverture télévisée du débat parlementaire constitue le quatrième point. Il s'agit également d'une question transversale puisque le profil des mandataires publics qui ont soulevé ce problème est très diversifié. Néanmoins, comparativement aux thèmes précédents, les lacunes identifiées en la matière n'ont été évoquées que par 30\% des répondants.

Enfin, qu'ils considèrent ou non qu'il existe des émissions politiques en Belgique, 20\% des interlocuteurs ont soulevé la question de la programmation des émissions Mise au point et Controverse. $25 \%$ de l'échantillon estime qu'il conviendrait de s'extirper des formats rigides qui président actuellement à la conduite et à la réalisation des émissions politiques existantes. Notons que cette classe de mandataires et celle considérant qu'il n'existe pas de réelle émission politique en Communauté française sont mutuellement exclusives : elles ne se recoupent pas.

\section{Les différences entre le public et le privé}

L'évaluation générale dressée ci-dessus doit être nuancée en fonction des divergences perçues entre les deux principaux éditeurs de services francophones. Les différences pointées quant à l'offre de programmes sont, il est vrai, relativement peu nombreuses. En effet, c'est dans le registre plus spécifique des genres télévisuels que les deux chaînes se différencient pour majeure partie. C'est pourquoi nous nous bornons à signaler que les mandataires interrogés dissocient les débats dominicaux principalement selon leur rythme et leur mise en scène.

Le rythme et la mise en scène sont en réalité symptomatiques d'un paramètre majeur discriminant l'offre de programmes: le statut de chaîne publique ou privée et l'obligation ou non de remplir des missions de service public. En réalité, les réflexions des représentants politiques se cristallisent autour de deux principes jugés antagonistes dans l'état actuel de l'offre : le «sérieux»/ la «mission» d'information et le dynamisme. D'où les réflexions soulevées par plusieurs interlocuteurs sur la manière de s'extraire des formats classiques. Malgré ces diffé- 
rences entre les chaînes, près de $40 \%$ des personnalités politiques interrogées pointent une tendance au nivellement dans la conduite des débats dominicaux. Enfin, notons que la question de l'empreinte du statut de la RTBF sur le contenu de ses programmes est posée à plusieurs reprises. Les critiques à cet égard se répartissent aisément suivant un clivage majorité-opposition à la Communauté française. Plusieurs parlementaires ont pointé ce qu'ils considèrent comme un manque d'impartialité non seulement dans le traitement de l'information politique mais aussi dans le traitement réservé aux invités au cours de certaines émissions.

\section{Le paysage audiovisuel extérieur}

Les paysages audiovisuels flamand et français semblent constituer, à de nombreux égards, une source d'inspiration dans la réflexion du personnel politique sur les équilibres à trouver au sein des émissions politiques francophones.

La référence du côté néerlandophone est l'émission Villa Politica diffusée sur la chaîne Een (VRT) et consacrée à la vie des hémicycles flamands et fédéraux. Elle est systématiquement citée par les représentants désireux de voir les débats parlementaires trouver une niche à part entière parmi la couverture de l'actualité politique en Communauté française. Dans une moindre mesure, les programmes Terzake et De zevende dag sont évoqués à titre exemplaire.

Dans le paysage audiovisuel français, l'émission Cent minutes pour convaincre de France 2 est placée aux antipodes des débats dominicaux belges francophones. Le nombre d'intervenants et le temps de parole imparti contribuent à en faire le débat d'idées tant attendu par les mandataires considérant qu'il n'existe pas de réelle émission politique sur nos chaînes. Les représentants politiques pointent encore les émissions France Europe Express et Culture et dépendances, ainsi que la brève émission quotidienne Les quatre vérités diffusée en début de matinée sur France 2.

En revanche, c'est également dans les champs de l'audiovisuel flamand et français que nombre de nos interlocuteurs identifient des mises en scène spectaculaires du politique et une perméabilité des frontières vie privée-vie publique plus poussée qu'en Communauté française. Dans le premier cas de figure, une émission est pointée régulièrement du doigt : De laatste show. Le mélange des invités auquel cette émission procède, les registres qui y sont abordés, entrecoupés d'interludes musicaux et de séquences de reportage au second degré, contri- 
buent à franchir un pas vers un mélange des genres - avancé - que tous les francophones n'acceptent pas. Les avis convergent pour nuancer cette perméabilité dans le sud de la Belgique où le représentant politique serait encore libre de doser ce qu'il souhaite ou non dévoiler. Mise en scène et porosité public-privé dans les émissions politiques posent la question des genres télévisuels.

\section{Le débat - talk-show : la polémique télévisuelle}

Polémique, conflit, conflictuel, agressif, chaos, foire d'empoigne, confrontation, provocation, affrontement, engueulade, bagarre, joutes et cacophonie : tels sont les qualificatifs attribués aux débats. Néanmoins, les interlocuteurs opèrent une distinction entre les deux débats dominicaux. Celle-ci est apparente lorsque l'on observe la distribution de ces termes par chaîne. En effet, ce registre d'altercations est généralement accolé à l'émission Controverse ou employé pour désigner un état d'esprit général dans ces débats. Peu d'occurrences sont associées à Mise au point. Les interlocuteurs tempèrent également leurs propos au sujet de la RTBF en précisant: «ça va encore » ou «c'est moins marqué », etc. Certains mandataires publics résument cette partition en parlant de «sensationnalisme » vs de «travail scientifique », d'autres parlent plus modérément d'approche «événementielle vs « approche positiviste».

Quoi qu'il en soit, le registre de la querelle traverse le corpus d'entretiens. L'énonciation dépasserait le registre de la contradiction, consistant à «apporter un point de vue contraire à un autre déjà exposé », pour s'inscrire pleinement dans celui de la polémique. Cette énonciation polémique serait mise en œuvre via plusieurs procédés :

Il faut qu'il se passe quelque chose, alors il y a les beaux mots, les phrases choc, les phrases slogan, les engueulades sur les plateaux... L'animateur qui renforce ça, quand quelqu'un commence à exprimer une idée de fond et que ça devient moins animé, moins vivant, l'animateur lui coupe la parole et passe au suivant.

On ressent la recherche du conflit dans le casting, on le ressent dans le scénario de l'ordre des questions, dans la manière dont ils les réinterpellent, ou ils passent au suivant, etc.

Certaines techniques seraient utilisées plus spécifiquement par l'animateur de la chaîne privée : «Vrebos met dans le ton de sa ques- 
tion une partie de la rage ou de l'impertinence du citoyen. Vrebos, il a un art pour faire monter la sauce, et pour poser des questions faussement nä̈ves. » Cependant, il semble que le climat général de «guerre verbale » dont nos interlocuteurs tentent une définition s'apparente davantage à une polémique de ton qu'à une polémique de fond basée sur un échange argumenté. En effet, elle est une pure monstration d'un affrontement.

Ce dissensus, manifestement présent sur les plateaux des deux principales chaînes francophones, si l'on en croit les interviewés, serait accru par une autre dimension de l'énonciation : la présence de témoignages. Ce que dénoncent un quart des mandataires interrogés, c'est l'instauration d'un témoignage particulier en archétype de la parole du citoyen. Dans la même veine, les interviewés s'interrogent sur la manière de trier les appels téléphoniques ou courriels diffusés à l'antenne et mettent en cause leur représentativité ou «valeur statistique ». Ces témoignages permettent dès lors de franchir un pas vers l'engagement de l'instance énonciatrice.

Le degré d'engagement constitue, en effet, le deuxième volet de l'examen de l'instance énonciatrice. En effet, ce thème a été abordé spontanément par 54\% des personnalités interviewées. Par engagement, nous entendons «le fait que l'énonciateur manifeste plus ou moins sa propre opinion ou ses propres appréciations dans l'analyse qu'il propose ou dans la façon de mettre l'événement en scène». La deuxième considération nous intéresse au premier chef puisque le dispositif conversationnel mis en œuvre par l'animateur (distribution de la parole et des interventions), sa composition des plateaux d'invités (choix des invités et priorité aux représentants des partis) et sa « starisation » constituent les principales références de notre corpus. Alors que les prises de positions partisanes constituaient l'un des griefs formulés à l'encontre de l'offre de programmes de la chaîne publique, l'engagement de l'instance énonciatrice semble viser au premier chef les représentations relatives à la chaîne privée.

D'un point de vue global, les interviewés affirmeront par exemple que «le rôle du journaliste est très important parce qu'il peut couper la parole à quelqu'un. Il peut, au contraire, laisser parler quelqu'un un peu plus longtemps. Il peut donner la parole d'abord à l'un, puis à l'autre. Donc, fatalement, son rôle n'est pas neutre ». D'un point de vue particulier ils préciseront, par exemple : «Vrebos arrête vraiment beaucoup plus vite les personnes et quelque fois il n'y a même pas l'expression d'une idée qu'il arrête. Le débat est tronqué. » 
Une différence assez nette entre les deux instances énonciatrices résiderait dans le processus de «starisation » ou de « vedettarisation » de l'un des animateurs. En réalité, il semble que cet engagement de l'instance énonciatrice aille de pair avec le processus de construction d'une énonciation polémique. Aux dires de nos interlocuteurs, le présentateur ne se borne pas à mettre la polémique en spectacle mais à assurer la polémique grâce à son opacité dans le débat.

Le regard critique posé par les personnalités politiques interviewées sur les plateaux des émissions dominicales pourrait s'expliquer, pour partie, en raison de l'orientation que prend la dimension pragmatique de la communication politique dans cet «espace de conflit». Cette recherche de «l'interaction agonistique» entre discours différents fonderait la relation entre l'émetteur-politique et le récepteur-téléspectateur sur un postulat de différence et non d'égalité.

\section{Les émissions de divertissement : l'homme ordinaire}

Les éditeurs de services procèdent parfois à l'invitation de représentants politiques à certaines émissions qui dépassent le cadre de représentation habituel du politique à la télévision. Jeux télévisés, variétés ou émissions spéciales à vocation non politique, nous les avons regroupés sous l'intitulé générique de «divertissement » en raison de la prépondérance de leur composante ludique. Nous avons demandé aux mandataires s'ils accepteraient de participer à une émission de divertissement. Les réponses obtenues ne sont pas en une seule teinte. Nous observons en effet une petite frange de NON catégoriques assortie d'une vaste série conditionnelle de «ça dépend ». Celle-ci est très hétérogène tant les opinions qu'elle recouvre s'apparentent parfois à des oui, parfois à des non. Près de $2 / 3$ des mandataires publics interviewés sont $a$ priori favorables à une participation. Observons les arguments avancés pour refuser une participation. D'abord, dans le registre des «non catégoriques »-qui totalisent 16.5 des $37.5 \%$ d'a priori défavorables - les arguments se partagent entre une dégradation de l'image de la classe politique et l'instauration d'une voie de garage pour la compréhension politique. Ensuite, dans le reste de la catégorie, la proportion d'inconvénients identifiés prend le pas sur les avantages.

Pour certains, dans la difficile équation qui préside à la décision, il convient de ménager les considérations éthiques (la personne comme substitut du programme) et les risques encourus (avoir l'air grotesque) avec les impératifs électoraux (tant au point de vue individuel qu'au 
point de vue collectif du parti). Ainsi, avoir un a priori défavorable ne signifie pas automatiquement refuser une invitation.

L'examen des a priori positifs à l'égard d'une participation mérite que l'on procède à un comptage des arguments. En effet, parmi les 26 arguments énoncés pour justifier leur concours à un programme de divertissement, les personnalités politiques interviewées usent à 12 reprises du registre des valeurs communes. Au premier chef apparaît «l'humain » associé aux vertus démocratiques. Bref, près d'un représentant sur deux, a priori favorable à une participation, invoque le registre de «l'homme-la femme ordinaire ». C'est le politique-miroir, humain comme tout le monde, «avec ses moments de joie et de désespoirs ». La dynamique identitaire nous semble pouvoir être assimilée à un processus de «projection-identification», d'autant plus que cet homme ordinaire parvient au récepteur par l'entremise de l'image.

Les $50 \%$ d'arguments qui ne puisent pas dans le registre des valeurs communes peuvent être résumés en trois axes. Tout d'abord, les programmes ludiques sont « grand public », y prendre part constitue donc une opportunité pour toucher un maximum de citoyens en une fois. Ensuite, ils permettent de se faire connaître auprès d'un public différent de celui qui suit les émissions politiques. Il y a donc un avantage en termes de notoriété : «la notoriété, ça fait partie de notre job, à tort ou à raison ». Enfin, le cadre détendu de ces émissions interagirait avec l'image de la personne pour la rendre sympathique. Il y a donc un bénéfice de popularité (comme pour le registre de «l'homme ordinaire $»$ du reste).

S'ils concèdent qu'il existe nombre d'avantages à se prêter au jeu des émissions ludiques, les représentants appartenant à cette catégorie n'en conditionnent pas moins leur participation à l'un ou l'autre de ces principes:

- Ne pas apparaître grotesque: «je travaille beaucoup, je fais mon travail sérieusement. Je ne vois pas pourquoi je mériterais d'être ridicule ». Néanmoins, tous les mandataires interrogés ne semblent pas placer la barre du ridicule au même niveau ;

- Pouvoir faire passer un message dans l'émission, être en mesure d'y « relayer certains combats »;

- Jouer le rôle du politique, c'est-à-dire ne pas être interchangeable avec n'importe quel citoyen présent sur le plateau ;

- Ne pas banaliser la fonction publique ; 
- Quant au registre de la vie privée, il s'agit d'une balise extrêmement élastique ;

- Enfin, cette participation ne peut pas devenir la règle.

Pour conclure cette section relative aux émissions de divertissement, tentons de dépeindre un tant soit peu ces deux chiffres impersonnels de $62.5 \%$ de mandataires a priori favorables et $37.5 \%$ a priori défavorables. Il semble ainsi intéressant d'étudier l'effet de la variable âge sur la constitution des a priori :

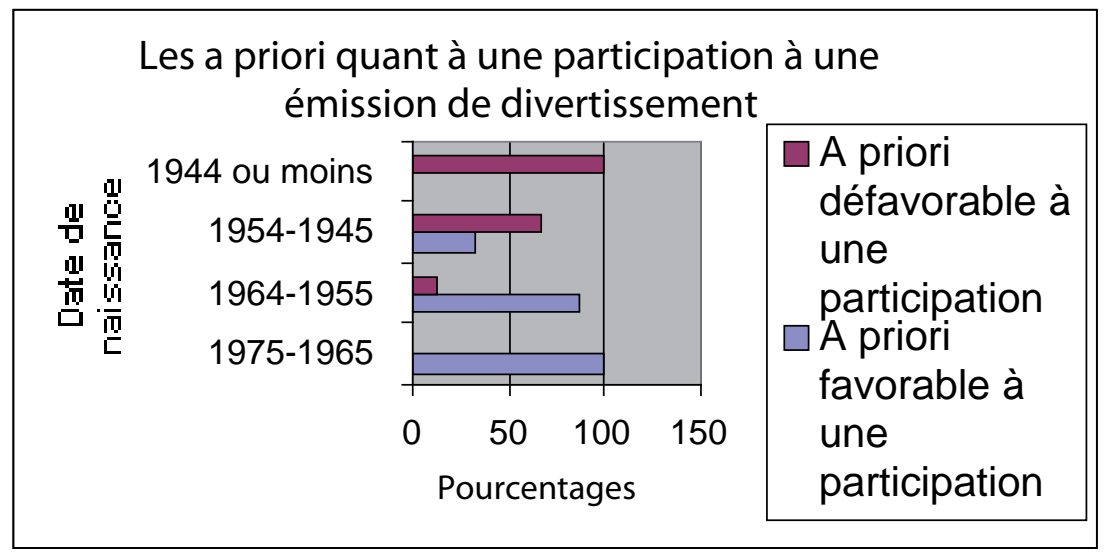

Il semble que l'année de naissance de nos interlocuteurs soit effectivement déterminante pour leur réponse. En effet, au plus ils sont âgés, au plus ils partent d'un a priori défavorable. Inversement, au plus ils sont jeunes, au plus ils partent dans un état d'esprit favorable. Ainsi, du point de vue des extrémités de classes, nous n'observons aucun a priori défavorables chez 30-40 ans contre aucun a priori favorable chez les plus de 61 ans.

\section{Emissions électorales et mélange des genres}

Nous avons interrogé nos interlocuteurs sur trois émissions diffusées au cours de la campagne électorale de 2004 : "Destination élections », «Pour qui voter ? » et « Face à l'électeur». Les avis sont tellement contrastés selon les émissions que l'on ne peut aborder le thème de l'interpénétration générique comme un bloc monolithique. 


\section{Destination élections et le mode authentifiant}

L'émission Destination élections relève d'une construction inspirée par les fictions télévisuelles. Pourtant, les représentations qu'en donnent nos interlocuteurs lui confèrent un important « effet d'authenticité ». Celui-ci peut s'expliquer par deux raisons. Premièrement, les invités sont confrontés à des situations de terrain choisies en fonction des dossiers qu'ils traitent. Deuxièmement, à de rares exceptions près, les hommes politiques rencontrent sur ce terrain des interlocuteurs anonymes qui leur font part de leurs remarques ou de leurs commentaires dans le feu de l'action. Ces interventions sont donc, en principe, non calculées et soumises à l'aléatoire, ce qui participerait au «naturel» de la situation. Ces deux critères apporteraient ainsi des gages d'authenticité à l'émission. Malgré la présence de caméras et le cadre particulier d'une campagne électorale, certains mandataires interviewés dépeignent «Destination élections » sous le mode quasi authentifiant, la présentant comme une découverte de «leur monde », scénarisée, certes, mais non simulée.

Les risques identifiés concernant une participation à cette émission résident précisément dans un glissement de l'émission du mode authentifiant au mode fictif. Ce glissement parait être en mesure de se produire dès lors que l'instance énonciatrice conserve exclusivement un certain type de passages de cette situation non simulée : «À certains moments, on pouvait être bien, pendant $95 \%$ du temps. Si vous dérapez $5 \%$, c'est les $5 \%$ de dérapage que l'on va retenir. Donc c'est particulièrement périlleux, surtout quand vous êtes exposés en situation réelle où vous pouvez avoir un zygomar ». En bref, c'est risqué.

\section{Pour qui voter? et Face à l'électeur: le mode ludique}

Les émissions Pour qui voter? et Face à l'électeur présentent un point commun : toutes deux recourent aux outils des jeux télévisés. Nous avons donc demandé à nos interlocuteurs s'ils étaient ou non favorables à l'introduction d'un aspect ludique dans une émission de campagne électorale. Les réponses enregistrées permettent de séparer l'échantillon en deux groupes :

\begin{tabular}{|l|c|c|}
\hline & Effectifs & Pourcentages \\
\hline Favorables & 12 & 50 \\
\hline Défavorables & 9 & 37.5 \\
\hline Non réponse & 3 & 12.5 \\
\hline
\end{tabular}


Les avis sont un peu plus mitigés quant à l'introduction d'un aspect ludique au sein de programmes politiques - qui plus est d'émissions électorales, identifiées comme le cœur des programmes politiques - que quant à une participation à un programme réellement ludique (divertissement). Pourquoi certain interlocuteurs sont-ils favorables à une interpénétration des genres ? Cette catégorie de mandataires publics énonce quatre types d'arguments : lutter contre l'ennui et le zapping, apprendre plus facilement, intéresser une plus grande frange de la population à la chose publique et forcer à la clarté du message. Une fois encore, c'est le registre des valeurs communes qui prévaut : réintégrer dans l'espace public les citoyens égarés. À cet égard, le principal grief formulé par l'autre catégorie d'interlocuteurs, défavorable au mélange ludique-politique, peut être interprété comme un brouillage de l'espace public. En effet, cette catégorie de mandataires considère qu'il ne faut pas faire jouer l'homme public-politique. Mixer le politique et le jeu, ce serait dévoyer le premier, le faire «sortir du chemin » qui est le sien. C'est précisément pour cette raison que près d'un tiers des mandataires favorables à une participation aux émissions de divertissement sont défavorables au mélange des genres. Dans le premier type de programme, l'homme politique n'insiste pas sur son caractère d'homme public, il est « comme tout le monde », avec ses forces et ses faiblesses. En revanche dans les émissions hybrides, le programme garde son caractère politique et le mandataire public y est présent en raison de son statut. Dès lors, c'est l'homme public que l'on fait jouer et non le citoyen, il y a un brouillage des repères dans l'espace public.

La participation aux émissions de divertissement pose pour certains une question de déontologie. Ils sont uniquement trois à avoir évoqué ce terme en tant que tel, mais il revient à d'autres moments dans les interviews. L'univers de représentations des transgressions journalistiques est focalisé sur le respect des personnes - respect de la vie privée. Néanmoins, des remarques relatives à la recherche de la vérité pointent en périphérie. Ainsi, certains mandataires associent les témoignages diffusés au cours des débats dominicaux à des généralisations abusives : "On tire d'une situation particulière des éléments de l'analyse d'une situation générale ». D'autres considèrent qu'il y a sur certains dossiers des failles dans la présentation des diverses facettes qui le composent.

Par ailleurs, $46 \%$ des politiques interrogés abordent la thématique du respect de la vie privée. Ces mandataires s'accordent pour dire que la perméabilité de la frontière vie privée / vie publique est assez limitée en Communauté française comparativement à la Flandre par exemple. 
En outre, certains distinguent le respect de la vie privée à la télévision de celui mis en œuvre par la presse magazine. Celle-ci transgresserait plus vite certaines limites au travers des reportages photos, par exemple. La frontière vie privée-vie publique est mouvante, elle dépend aussi de ce que le personnel politique veut bien donner à voir au téléspectateur. Tous ne fixent pas les limites au même seuil.

La déontologie politique pose clairement, dans les représentations de nos interlocuteurs, la question de la mise en scène du politique par le politique lui même. Elle renvoie non seulement à la personnalisation de la communication politique en découvrant une part de sa vie privée, aux disputes ou règlements de compte via les médias ou encore à l'instrumentalisation de la télévision par le personnel politique. Les phénomènes identifiés participent en fait à la construction d'un ethos télévisuel. Cet ethos, c'est l'image que l'orateur donne de lui-même auprès du public. Lorsqu'il prend la posture de l'homme ordinaire ou découvre sa vie privée, le représentant politique projette une identité dans un champ social déterminé, il ne laisse pas s'échapper son identité. D'où l'importance des stratégies de communication.

\section{Perspective diachronique}

La mise en œuvre de ces stratégies communicationnelles a introduit des évolutions dans les modes de présentation du politique à la télévision, et notamment dans les discours que tiennent les mandataires publics devant les caméras. Parmi les évolutions décrites, $63.2 \%$ sont focalisées sur l'image et la mise en scène du discours. En effet, d'une part, le fond de la communication politique serait relégué à l'arrièreplan de l'impression générale laissée au téléspectateur-citoyen et de la nécessité d'apparaître à l'écran. D'autre part, chaque apparition télévisée serait minutieusement intégrée dans des scénarios préétablis et ne laisserait plus de place pour la spontanéité.

Le reste des variations observées dans le discours politique peut être synthétisé comme suit : certains affirment que la prégnance de la communication politique «scientifiquement étudiée » a aseptisé le discours et contribué à faire disparaître les affrontements idéologiques. D'autres considèrent que la communication se professionnalise pour être en mesure de répondre aux exigences médiatiques. Enfin, les deux dernières évolutions concernent davantage les personnes que le discours. La première met en évidence l'inégalité de fait entre les légitimités élective et cathodique : "J'ai connu des mandataires qui 
étaient d'excellents travailleurs de fond mais qui étaient de mauvais orateurs et qui ne se sont jamais fait une notoriété ». Dans le même ordre d'idées, il peut y avoir un décalage entre les légitimités cathodique et décisionnelle : "Vous avez de plus en plus de ministres qui sont là pour la galerie. À la rigueur ils sont sympas, donc ils font des voix, donc on les nomme; vous avez ma parole d'honneur qu'ils ne décident rien $»$.

En outre, il semble intéressant d'observer quel type de mandataire public se désole de voir le discours de fond de la classe politique relégué derrière l'image et la mise en scène. En procédant à un croisement de cette variable avec celle des a priori quant à une participation aux émissions de divertissement, $75 \%$ des mandataires publics « dénonçant » un discours politique de plus en plus axé sur l'image et la mise en scène se montrent pourtant a priori favorables à une participation aux émissions de divertissement. Or, lorsqu'ils se présentent comme des hommes ou des femmes ordinaires dans ces programmes, ils donnent à voir une image d'eux-mêmes qui est minutieusement contrôlée.

\section{Le visage du récepteur}

Les informations que les mandataires publics reçoivent en retour de ces prestations télévisées sont variables selon le profil sociologique des récipiendaires du message. En effet, la rétention du fond du message augmenterait avec le degré de conscientisation politique - et d'intérêt porté au sujet traité. Les commentaires en provenance du grand public (par e-mail, par courrier, en rue, etc.) sont donc essentiellement formels. C'est en tout cas ce qu'affirment $62.5 \%$ de nos interlocuteurs.

Si l'information de retour provient surtout sur les aspects formels, il faut s'interroger sur la rétention du contenu. Voici les réponses obtenues:

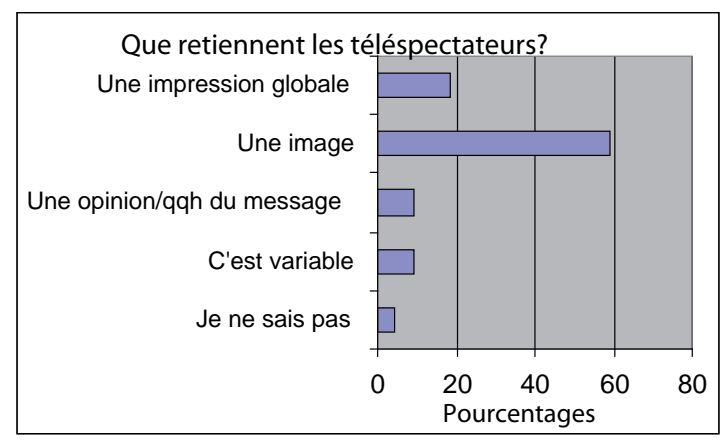


59\% des interviewés penchent en faveur de la rétention du montré plutôt que du dit. A priori, ce chiffre pourrait témoigner de ce que les praticiens du marketing politique appellent «une perte d'effectivité $d u$ message ». Celle-ci se fait, semble-t-il, au détriment du fond, mais elle laisse la porte ouverte à la construction d'un ethos télévisuel.

\section{Analyse des questionnaires aux parlementaires}

Si le regard porté par les représentants du pouvoir législatif est susceptible d'être différent de celui des personnalités politiques interrogées en face à face, c'est peut-être en raison de leur degré d'exposition aux caméras. Parmi les députés communautaires. 38\% d'entre eux n'ont pris part à aucune émission au cours de la période étudiée. Qui plus est, la catégorie des parlementaires de la Communauté française est relativement hétérogène : $71 \%$ des répondants totalisent de zéro à trois passages télévisés en deux ans, alors qu'une frange de $29 \%$ a été exposée entre quatre et neuf fois aux caméras sur cette même période.

Notons encore que le taux de participation aux émissions télévisées doit être appréhendé dans le contexte temporel visé par le questionnaire. En effet, ce dernier inclut une période de campagne électorale au niveau des entités fédérées, ce qui augmente sans aucun doute le « taux de médiatisation » des députés : $40 \%$ des raisons invoquées pour motiver une participation se rapportent à la campagne électorale. En d'autre temps, le taux de $38 \%$ de non-participation pourrait être plus élevé.

Enfin, les canaux au travers desquels les députés véhiculent leur communication divergent aussi de notre groupe d'interlocuteurs précédent : les parlementaires de la Communauté française sont exposés au premier chef (pour 43\%) aux caméras des télévisions locales.

\section{L'univers de représentation du politique à la télévision}

Une première lecture des résultats met en exergue que les répondants éludent le temps électoral et se focalisent exclusivement sur le temps «usuel» de représentation du politique. En effet, près de la moitié des définitions collectées délimitent l'univers des programmes politique a contrario. Cela signifie que leurs énonciateurs définissent une émission politique en opposition à ce qui se fait actuellement le dimanche sur RTL et sur la RTBF. En voici une brève synthèse. Une émission politique est : 
- un débat sérieux, évitant le sensationnalisme ;

- un débat politique avec un animateur à la hauteur ;

- un débat avec un animateur sans appartenance politique ;

- une émission qui ne débouche pas sur une épreuve de force, une confrontation de partis ;

- une émission où chacun peut s'exprimer sans être interrompu à tout moment ;

- une émission diffusée à une heure de grande écoute.

À ce premier niveau définitionnel, constitué des griefs formulés à l'encontre des programmes offerts, vient se greffer une pluralité de définitions qui recoupent celles énumérées au cours des face-à-face. Le débat contradictoire, démocratique, de société, bref, le débat d'idées demeure toujours en bonne place. Il est accompagné du positionnement partisan. En revanche, ce qui constitue un trait original dans les définitions fournies par les élus communautaires, c'est la référence récurrente au citoyen ainsi qu'à la vie de la cité. Si l'on y ajoute les définitions presque tautologiques selon lesquelles une émission politique traite d'un dossier politique ou relate un fait politique, nous sommes en mesure d'affirmer que les parlementaires ramènent au centre de l'univers de représentation du politique ce que la majorité des personnalités politiques avaient placé en périphérie. Ce faisant, ils élargissent le champ du politique à la télévision.

En dépit de cet élargissement, le nombre d'émissions remplissant ces critères définitionnels et méritant par voie de conséquence le qualificatif de «politique » se voit diminué. En effet, si 62,8\% des mandataires publics que nous avons interrogés en face-à-face qualifiaient Controverse de « politique » après avoir fourni une définition de ce type d'émission, ils ne sont plus que $33 \%$ du côté des députés communautaires qui ont répondu par questionnaire. Il semble que les « définitions a contrario $»$ y soient pour beaucoup dans le faible pourcentage obtenu. L'émission Mise au point, quant à elle, obtient un pourcentage à peu près semblable à celui acquis précédemment : $62 \%$ contre 66,6 . Il en va de même pour les J.T. En revanche, l'émission L'invité est évoquée nettement moins spontanément.

\section{Caractéristiques idéales d'une émission politique}

À la question «Quelles seraient, selon-vous, les caractéristiques idéales d'une émission politique à la télévision? », les parlementaires 
répondent par des critères de fond et de forme. Ceux-ci prennent non seulement le contre-pied de ce qui est proposé par les programmes dominicaux de RTL et de la RTBF, mais ils fédèrent également les principaux points forts attribués aux programmes proposés à l'extérieur de la Communauté française. En effet, les émissions Cent minutes pour convaincre et France Europe Express sont, une fois encore, érigées en « modèles » à suivre pour des raisons inhérentes à la qualité des journalistes-animateurs, à la conduite et à la structuration du débat, à la composition des plateaux, à la sélection des sujets, à la programmation et enfin, pour des raisons d' " attractivité » du public.

Du point de vue des qualités de l'animateur de débat, deux tiers des participants ont pointé la nécessité d'être en présence d'un journaliste bien documenté, impartial et pas trop intervenant. La question de la partialité des animateurs des chaînes belges francophones constituait déjà une thématique récurrente dans les propos des personnalités politiques médiatiques. Ils étaient $54 \%$ à dénoncer une manifestation de l'engagement de l'instance énonciatrice dans l'analyse qu'elle propose ou dans la manière de mettre en scène l'événement. Ils sont ici $30 \%$ à énoncer explicitement qu'une bonne émission politique doit obligatoirement comporter un animateur neutre.

Concernant la conduite et la structuration du débat, l'émission politique idéale ne devrait pas verser dans le «sensationnalisme», la «politique-spectacle » voire le «populisme», caractéristiques énoncées par certains en vue de marquer une différence avec les programmes proposés en Belgique francophone. Gravitent autour de cette recherche de sensationnel, la question de l'équilibre et du respect du temps de parole imparti à chaque intervenant, mais aussi celle du mimétisme des chaînes de télévision. Nous touchons ici à la thématique d'un éventuel nivellement dans la conduite des débats dominicaux telle que soulevée lors des entretiens. Si les programmes Cent minutes pour convaincre et France Europe Express sont érigés en types idéaux, c'est principalement en raison de la longueur du temps de parole qu'ils attribuent aux intervenants, ce qui permettrait non seulement d'éviter «les foires d'empoignes » mais aussi d'aborder les sujets en "profondeur», de développer un «discours structuré » ou plus généralement de mettre en ouvre un «véritable débat d'idées ». Enfin, un plateau d'invités reflétant un pluralisme politique et une programmation à une heure de grande écoute constituent les derniers critères formels caractérisant une émission politique idéale. 
Au vu de ces caractéristiques, il semble qu'en dépit de leur moindre médiatisation, les députés communautaires posent des critères d'évaluation des émissions politiques proposées en Communauté française analogues à notre premier groupe d'interlocuteurs et usent des mêmes modèles de référence pour les apprécier.

\section{La présence des politiques à la télévision}

Si les deux groupes se différencient fort peu dans l'appréciation des programmes offerts, ils ne se distinguent pas plus concernant la question de la présence, suffisante ou non, des représentants politiques à la télévision. En effet, dans chaque catégorie de mandataires, une majorité de répondants considère que les représentants politiques sont suffisamment présents à l'écran. Le taux de médiatisation plus faible des parlementaires de la Communauté française laissait penser a priori que leur avis aurait été plus nuancé que celui émis par les personnalités politiques médiatiques.

Le « oui » des politiques médiatisés et des députés communautaires se différencie néanmoins sur un point. Dans le premier cas de figure, le « oui » est davantage quantitatif que qualitatif. Quantitativement, les représentants politiques sont suffisamment présents. Ce qui pose question, c'est davantage la qualité de ce qui est donné au téléspectateur, c'est-à-dire la qualité des interventions et des lieux d'expression de la classe politique (pas de grand débat d'idées). Dans le second cas de figure, les députés communautaires s'accordent à dire que la classe politique est suffisamment présente à l'écran. Néanmoins, ils s'interrogent non seulement sur la qualité mais aussi sur la répartition de cette exposition médiatique entre les différents représentants politiques : ce sont «toujours les mêmes sur les deux chaînes en Communauté française ».

Nous avons soumis onze propositions aux parlementaires au sujet de l'action de la télévision sur la chose publique et sur les représentants politiques. Les réponses mettent au jour une disparité entre le message formel et le fondamental. Les seules actions de fond de la télévision consisteraient à mettre en scène le débat politique et partant, à permettre la compréhension de l'actualité politique auprès du téléspectateur. Cette actualité ne serait pas n'importe laquelle, puisque celle relative à la Communauté française, et plus précisément à son instance législative, serait très insuffisamment traitée par la télévision belge. En outre, si le médium télévisuel insère le débat politique sur la scène publique, il 
n'en refléterait pas les différents éléments et ne donnerait pas toujours une image correcte de cette vie politique. Aux dires de certains députés, la télévision ne présenterait que les aspects négatifs de la vie politique, d'autres considèrent que le climat sensationnaliste d'empoignades sur les plateaux de télévision ne donne pas une image « reluisante» de la vie politique. Enfin, quelques parlementaires estiment que l'image des mandataires publics véhiculée par la télévision tend à les abstraire de la population.

L'action de la télévision serait essentiellement concentrée sur les aspects formels : elle valorise les personnes et crée des personnages, elle s'attache en premier lieu à l'image et dans certains cas, ridiculise le politique. Les trois premières caractéristiques renvoient à une « personnalisation » du personnel politique via le médium télévisuel. Dans les représentations de $62 \%$ des députés communautaires, la télévision œuvre à une personnalisation élevée du personnel politique. Elle valorise leur personne, comme s'il était possible de s'extraire des personnalités - face sociale et contrôlée de la personne - et aboutit à créer des personnages. La télévision valorise les images, ce qui permet de franchir un pas vers la construction d'un ethos télévisuel. Les députés qui ont répondu au questionnaire se montrent très sceptiques à l'égard de cette personnalisation. En effet, 95\% d'entre eux se disent défavorables tant au fait de s'attacher en premier lieu à l'image du politique qu'à celui de créer des personnages. Les avis sont plus nuancés du point de vue de la valorisation des personnes, puisque 52,5\% des répondants y sont défavorables alors que $38 \%$ considèrent qu'il s'agit d'une bonne chose.

\section{Les émissions de divertissement}

Environ $30 \%$ des personnalités politiques médiatiques que nous avons rencontrées ont pris part à des émissions de divertissement; par contre, seulement $5 \%$ des répondants au questionnaire a participé à ce genre de programme. En fait, tous les autres n'ont jamais été invités dans de telles émissions dépassant le cadre politique au sens strict. Les parlementaires se montrent pourtant moins sceptiques à l'égard d'une éventuelle participation. La moitié de tous ceux qui n'ont jamais été invités répondent par un «oui » franc à la question : «Si vous l'aviez été, y auriez-vous participé ?». Un quart d'entre eux réservent leur réponse selon la nature de l'émission de divertissement à laquelle ils seraient invités - «tout dépend de l'émission ». Enfin, le dernier quart 
de répondants rétorque un «non » catégorique à notre question. Ici, l'âge des répondants ne semble plus avoir de prise sur les modalités de la réponse, contrairement aux personnalités politiques médiatiques. Il existe un «nivellement » en faveur d'une participation. Notons que la moindre exposition des députés communautaires aux caméras ne nous semble pas constituer un facteur potentiellement explicatif de ce phénomène. En effet, plusieurs participants qui n'ont effectué aucun passage télé en deux ans réservent leur réponse ou refusent purement et simplement l'éventualité d'une participation. Inversement, nombre de mandataires qui enregistrent à leur actif quatre à neuf passages télévisés au cours des deux dernières années répondent par un « oui » franc à la question de savoir si oui ou non ils accepteraient de prendre part à ce type de programme. Peut-être la volonté de bâtir une communication plus personnelle avec le téléspectateur-citoyen est-elle davantage marquée chez ces élus !

$\mathrm{Au}$ vu de ces analyses, il semble que le degré moindre d'exposition aux caméras de notre seconde catégorie de personnel politique ne modifie pas en profondeur leur appréhension du traitement médiatique de la vie politique. Si les processus évolutifs dépeints peuvent s'apparenter à un phénomène de mise en scène, celle-ci se fixerait sur les émissions qui a priori relèvent du genre politique au sens strict - les débats dominicaux - et sur certaines caractéristiques intrinsèques et fondatrices du traitement médiatique de l'actualité. En revanche, elle n'empêcherait pas, pour la majeure partie des représentants politiques, leur concours aux émissions qui sortent du cadre politique. 
